\title{
Derecho a la intimidad y redes sociales: relación
}

\section{Right to privacy and social networks: relationship}

\section{Yenifer Beleño Casarrubia', Melissa Sahara Caballero Paternina², Ligia Rosa Martínez Bula³.}

${ }^{1}$ Docente Tecnología e informática, Institución Educativa Lacides C. Bersal, Secretaria de educación de Lorica - Córdoba, Colombia.

Yenic1987@gmail.com

2 Docente básica primaria, Institución Educativa San Anterito, Secretaria de educación de Montería- Córdoba - Colombia.. sahara.chevalier@gmail.com

${ }^{3}$ Docente de inglés, Corporación universitaria del caribe CECAR, Sincelejo- Colombia ligia.martinezbu@cecar.edu.co

Recibido: 15/ago/2020 - Revisado: 30/sep/2020

Aceptado: 30/oct/2020 - Publicado: 30/dic/2020

Resumen Este es el resultado de una investigación que tuvo como objetivo identificar las concepciones de derecho a la intimidad que tienen los estudiantes de Lic. Informática de la Universidad de Córdoba y su relación con el uso de la red social Facebook. Se trata de un estudio cualitativo, basado en la etnografía educativa como estrategia metodológica. Se aplicaron técnicas como la entrevista semiestructurada y la observación no participante para la recolección de los datos, que posteriormente fueron analizados a partir de la categorización de sus contenidos.

Palabras claves concepciones, derecho a la intimidad; intimidad; redes sociales; Facebook.

Abstract This is the result of an investigation that aimed to identify the conceptions of the right to privacy held by students of Computer Science Degree at the University of Córdoba and their relationship with the use of the social network Facebook. It is a qualitative study, based on educational ethnography as a methodological strategy. Techniques such as the semi-structured interview and non-participant observation were applied to collect the data, which were subsequently analyzed from the categorization of their contents.

Keywords conceptions; right to privacy; privacy; social networks; Facebook. 


\section{Introducción}

Las investigaciones relacionadas con el uso de las nuevas tecnologías y prácticas dentro de las redes sociales en relación con el cuidado de la intimidad y la privacidad por parte de los jóvenes, se ha convertido en un tema de interés para los investigadores (Rodriguez \& Magdalena, 2016), (Romero \& Herrero, 2015), (Arteaga \& Ayala, 2015), (Linne, 2016), (Durán, 2014), (Tello, 2013). En esta misma línea los trabajos destinados al estudio de la intimidad y las redes sociales han abordado esta temática desde la perspectiva de los Derechos Humanos, es decir, se ha estudiado el uso de las redes sociales en relación con el Derecho a la intimidad (Salazar, 2014), (Ferreyra \& Baquero, 2014). Las practicas dentro de la web y específicamente las redes sociales, son un campo de estudio muy común en la actualidad, no obstante, son pocas las investigaciones realizadas basándose en los Derechos Humanos dentro de estos ambientes virtuales y menor aun el número de trabajos realizados con estudiantes de Licenciatura en informática, futuros docentes de tecnología y alfabetizadores en medios.

Por lo anterior, se consideró relevante identificar y describir cuáles son las concepciones de derecho a la intimidad que tienen estos jóvenes y contrastar dichas concepciones con sus prácticas dentro de las redes sociales en relación a este derecho; desde el punto de vista de las teorías cognitivas, el desarrollo y resultados de este estudio son pertinentes, dado que al conocer las concepciones de los estudiantes sobre el derecho a la intimidad en las redes sociales y la manera como éstas se reflejan en su práctica, se ajustan a la idea de que tales concepciones son representaciones mentales que hacen parte de la estructura de pensamiento del sujeto; quien por lo general las desconoce, porque están arraigadas en lo más profundo de su estructura cognitiva, al ser producto por un lado, de su experiencia y por otra parte, de su formación académica y social; (Thompson, 1992) considera las concepciones como una estructura mental general, que encierra creencias, significados, conceptos, proposiciones, imágenes mentales y preferencias".

Este tipo de estudios resulta apropiado para generar estrategias que permitan evidenciar las desventajas y riesgos que existen en el ciber-espacio, a los que están expuestos los usuarios de las redes sociales; abordar esta problemática desde la perspectiva del derecho a la intimidad, aporta al conocimiento y reconocimiento de los derechos humanos, especialmente el derecho a la intimidad, el cual es ignorado y desconocido por algunas personas, el desconocimiento de los derechos dificulta la defensa de estos; además, servirá como base para llevar a cabo futuras investigaciones relacionadas con el objeto de estudio del presente trabajo.

\section{Marco metodológico}

Surge la pregunta de investigación enmarcada con el objetivo general planteado en el presente estudio: ¿Cuál es la concepción que los estudiantes de la Licenciatura en Informática de la Universidad de Córdoba tienen acerca del derecho a la intimidad y su relación con el uso de las redes sociales: caso Facebook?

\subsection{Contexto y participantes}

El proceso de selección de los estudiantes, fue realizado mediante un muestreo intencionado o de conveniencia, que como cualquier estrategia de selección intencional o deliberada, requirió del desarrollo de un perfil de los atributos esenciales que debía cumplir cada estudiante que haría parte de la muestra; la definición de los atributos por parte de los investigadores, tuvo un carácter claramente selectivo o diferenciador del sujeto seleccionado, es decir, dicho sujeto debía cumplir con los requisitos exigidos por los investigadores, para de esta manera estudiar el caso de forma eficaz y óptima. En el proceso de selección de los atributos que debía cumplir cada estudiante, se definió que los estudiantes debían estar cursando 
tercer semestre de Licenciatura en Informática en la Universidad de Córdoba, durante el segundo periodo académico del año 2017, debido que a partir del tercer semestre, disminuyen los casos de deserción estudiantil, impidiendo así la continuidad en el proceso. Además de poseer una cuenta de Facebook, el estudiante adicionalmente debía comprometerse a confirmar a los investigadores como amigos dentro de su red social Facebook y permitir la observación de sus perfiles, para posteriormente ser estudiados $y$ analizados, asimismo se requería que el participante estuviera interesado en el estudio, contara con buena actitud para proporcionar la información requerida y también tuviera el tiempo libre suficiente para recoger y transmitir información.

\subsection{Procedimiento}

Se aplicó a los estudiantes una entrevista semiestructurada con el fin de identificar las concepciones y conocimientos que ellos tenían acerca del derecho a la intimidad y la forma como la cuidaban y protegían en las redes sociales. Las entrevistas fueron transcritas en diferentes textos, que luego fueron codificados de manera correlativa, a través de un método de investigación cualitativo. Después del tratamiento informático de los textos mediante el programa de análisis de datos cualitativo Atlas.ti (v.7.), se establecieron un serie de categorías y subcategorías; la primera correspondía al concepto de intimidad que tenían los estudiantes y a los aspectos que ellos consideraban como íntimos, porque se consideró necesario conocer primero cuál era la definición de intimidad que ellos manejaban, para seguidamente indagar acerca del derecho a la intimidad, término que abarca una definición más compleja. La segunda categoría de análisis correspondió al derecho a la intimidad, considerada la más importante dentro del proceso investigativo; en este punto se analizó el nivel de conocimiento que los estudiantes tenían acerca del derecho a la intimidad y a la normatividad nacional $e$ internacional que garantiza la protección y defensa de este derecho. La tercera categoría estaba relacionada con las concepciones de derecho a la intimidad que tenían los participantes; en este punto se buscó identificar y describir cuáles eras dichas concepciones y que tan cerca estaban de las definiciones establecidas por los teóricos y normas. La cuarta categoría correspondía a las redes sociales, en ella se realizó el análisis de la información consignada en los formatos de observación, para conocer los contenidos compartidos por los participantes y las prácticas de éstos dentro de la red social Facebook y por último la quinta categoría correspondiente a intimidad y tics, cuyo objetivo era conocer la relación existente entre la concepción de derecho a la intimidad de los estudiantes de informática y el uso que ellos hacen de la red social Facebook.

\section{Cuáles son las concepciones de derecho a la intimidad, que tienen los estudiantes y su relación con el uso de la red social Facebook}

Durante el análisis se transcriben los comentarios de los/las entrevistados/as, clasificados así: EP (Entrevista participante), el número de entrevista a la que corresponde. Así como $\mathrm{M} \mathrm{o} \mathrm{H} \mathrm{(Hombre} \mathrm{o} \mathrm{Mujer)}$ para identificar el género de la persona a la que ha sido aplicada la entrevista. Se reserva la información personal de los entrevistados de acuerdo con la fundamentación y diseño metodológico.

Durante el análisis se realizó una observación a los perfiles de Facebook de los participantes y se transcribieron los comentarios de las investigadoras en un formato de observación, identificando con las letras FO (Formato de Observación) y el número del formato al que corresponde. Dado que el objetivo era conocer las concepciones de derecho a la intimidad que tenían los participantes, se consideró pertinente primero indagar acerca de lo que para ellos es la intimidad.

Teniendo en cuenta el análisis que se realizó a las respuestas suministradas por los estudiantes durante la aplicación de la entrevista, se pudo observar en los hallazgos obtenidos, que los estudiantes tienen conocimientos básicos sobre lo que es la intimidad, basados en la experiencia y en ideas que han desarrollado desde lo empírico, al analizar las 
respuestas obtenidas, se pudo observar que para algunos de ellos la intimidad es toda aquella información, acciones y datos relacionados con lo familiar, lo laboral y las amistades; las respuestas más relevantes dentro del campo de investigación dadas por los estudiantes a la hora de definir intimidad y mencionar que aspectos hacen parte de esta fueron:

EP1H. "Los aspectos que hacen parte de mi vida íntima son: mi familia, mi trabajo, mis amigos, hacen parte de mi vida íntima y mis clientes"

EP6M. "La familia, las relaciones que tengo con diferentes personas, mis proyectos"

EP8M. "Las cuestiones familiares"

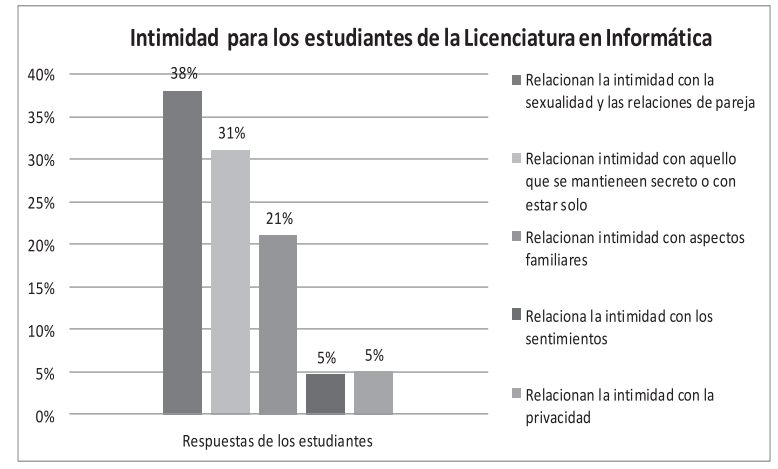

Figura 1. Concepciones de Intimidad.

Mientras que para otros, intimidad es aquella información o aquellas acciones que se tienen o se hacen en secreto, lejos de la vista de los demás, este grupo no considera que la familia y los amigos hacen parte o deban tener acceso a su intimidad, sino que la intimidad debe guardarse y conservarse para "uno mismo", por ejemplo, algunos contestaron que consideran que la intimidad no puede ser vista u observada por nadie más "Yo Considero que la intimidad es esa parte que sólo puede ver uno y que nadie más puede ver u observar"(EP4H.); o explican que la intimidad son aquellas cosas que mantienen para sí mismos u ocultas "Cuando se habla de intimidad pienso que es algo sobre uno mismo algo muy secreto, oculto, de ti mismo" (EP11M).

Para otros, Sin embargo, la intimidad es estar solos, tener un espacio o lugar en el cual estar en soledad y que dicho espacio sea respetado, también ven la intimidad como aquello que se tiene o se hace lejos de la vista de los demás. Afirman que intimidad es "Un ambiente donde estoy yo únicamente" (EP3H).

No obstante, a pesar de las diferencias encontradas en la concepción de intimidad que tienen los jóvenes estudiantes de la Licenciatura en Informática, los participantes comparten la idea de que los aspectos de su vida pertenecientes a la intimidad son aquellos concernientes a las relaciones de pareja y la sexualidad en general.

EP5M. "Un ejemplo de algo íntimo para mí, es cuando alguien está con su pareja".

EP4H. "De mi vida íntima hacen parte la sexualidad, mis relaciones sexuales, mi inclinación sexual, mi forma de vestir mi relación con mi pareja".

EP11M. "La intimidad en este caso no tengo novio si tuviera novio o alguna persona con la que salga".

$\mathrm{PE} 13 \mathrm{H}$. "Son aquellas conversaciones que tengo con mis papás acerca de las relaciones sexuales y cosas asi".

También, cabe mencionar que entre los hallazgos se encontró que los estudiantes tuvieron muchos aciertos a la hora de definir la intimidad y mencionar los aspectos de la vida que pueden ser considerados como íntimos.

EP7H. "Para mí los aspectos que hacen parte de la vida íntima serían como las experiencias y vivencias que tengo cada día".

EP8M. "Es nuestra información, pues la que no queremos que los demás conozcan de nosotros".

Por último, es importante mencionar un aspecto curioso encontrado en las respuestas de los estudiantes, se observó que relacionan el concepto de intimidad con el de privacidad, es más, los utilizan como sinónimos.

EP6M. "La intimidad es algo muy personal de cada persona, es algo muy privado".

Luego de cuestionar a los estudiantes acerca de lo que era para ellos intimidad, se procedió a indagar por lo que era para ellos el derecho a la intimidad y la información concerniente a éste. Con base en las respuestas dadas por los estudiantes en las entrevistas, se identificaron dichas concepciones y resulta curioso que aunque tienen una idea bastante cercana y acertada a la hora de definir o explicar en 
qué consiste el derecho a la intimidad, presentaron dudas y alegaron desconocimiento, por ejemplo, los participantes afirman que no habían considerado a la intimidad como un derecho "Realmente nunca he mirado la intimidad como un derecho" (EP1H).

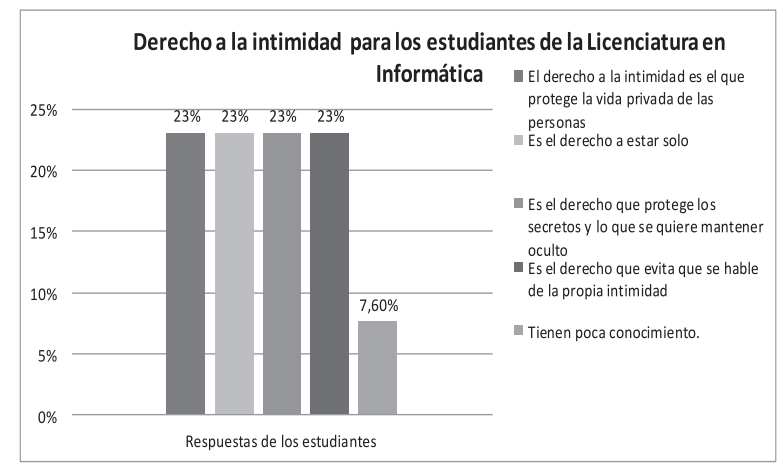

Figura 2. Concepciones de derecho a la intimidad.

Sin embargo, tienen aciertos a la hora de definir lo que es el Derecho a la Intimidad y lo que este incluye, puesto que, cuando se les pidió que expusieran que era para ellos el derecho a la intimidad, éstos respondieron:

EP3H. "El derecho que tengo a guardar cosas muy personales".

EP5M. "Para mí es el derecho que yo tengo de guardarme cosas que no quiero mostrarle a nadie más".

EP7H. "Derecho a la intimidad vienen siendo las leyes que garantizan que una persona tenga y se le proteja su propio espacio".

Se puede afirmar, que tienen bastante idea de lo que es la intimidad y de los aspectos que hacen parte de ésta y basados en este concepto han creado su concepción de Derecho a la Intimidad, aunque al definir dicho derecho reiteran en el error de confundir intimidad con privacidad, sin mencionar o destacar las diferencias existentes entre ambos términos.

$E P 2 H$. "El derecho a la intimidad es como el derecho a conservar mi propia privacidad".

EP1H. "Lo relaciono más que todo con privacidad".

Resulta curioso descubrir que, a pesar de que pueden decir en qué consiste el derecho a la intimidad, los participantes manifiestan desconocimiento de las leyes existentes a nivel nacional e internacional que protegen este derecho fundamental.

EP1H. "Leyes como tal, no sé cuáles son las que protegen el derecho a la intimidad, ni en Colombia ni en el mundo".

EP13H. "No sé, no tengo conocimiento de ninguna de ley".

$Y$ quienes manifiestan tener conocimiento de la existencia de estas leyes, señalan no haberlas leído o no conocerlas

EP5M. "Si existen para mí leyes que protejan la intimidad, pero no las conozco directamente".

EP6M. "No tengo un conocimiento exacto de las leyes que protegen el derecho a la intimidad, pero sí sé que existen".

EP8M. "Sí sé que existen, pero no las he leído".

Los entrevistados explican y dan a conocer las razones por las cuales no tienen conocimiento de las leyes que protegen el derecho a la intimidad. Algunos opinan que las desconocen por falta de iniciativa propia y desinterés en el tema.

EP8M. "Porque no me ha dado la curiosidad de buscar y leer".

EP10H. "No las conozco creo que por pereza de leer acerca de eso".

Por otro lado, otros estudiantes manifiestan que la información relacionada con el derecho y las leyes que lo protegen, no se encuentran disponibles y no hicieron parte de los contenidos estudiados en la escuela y/o universidad, además mencionan que los padres no les han brindado este tipo de información.

EP1H. "No estamos pensando o inculcando esas leyes y nuestros padres tampoco nos enseña y el colegio menos".

$\mathrm{EP} 2 \mathrm{H}$. "De pronto las desconozco porque no hay suficiente información".

En lo que están de acuerdo los participantes, es en que no conocen las leyes que protegen el derecho a la intimidad a nivel nacional o internacional y no han buscado información al respecto porque no se han visto en la necesidad de hacerlo, ya que según ellos comentan, no han sufrido abusos ni violaciones a este derecho.

EP6M. "Creo que las desconozco porque no me he tomado el tiempo de ir a investigar sobre este tipo de 
leyes, además no me he visto en una situación donde sea necesario velar por mi derecho a utilizar las leyes de este tipo".

$\mathrm{EP} 13 \mathrm{H}$. "Porque personalmente no me ha pasado ningún caso y no me ha tocado investigar".

En este punto es interesante mencionar que el pensum de la Licenciatura en Informática tiene un módulo con contenidos relacionados a las leyes existentes a nivel nacional, pero se refieren más a las leyes de tipo educativo, haciendo énfasis en la Ley General de Educación, un módulo ofrecido a los estudiantes de los programas de la facultad de educación de la Universidad de Córdoba.

Sin embargo, y a pesar de no conocer las leyes que protegen el derecho a la intimidad y de no considerar la intimidad como un derecho fundamental, los estudiantes aciertan a la hora de reconocer $y$ mencionar algunas vulneraciones a este derecho, relacionándolo con injerencias a su vida sexual o cuestionamientos referentes a su ropa interior.

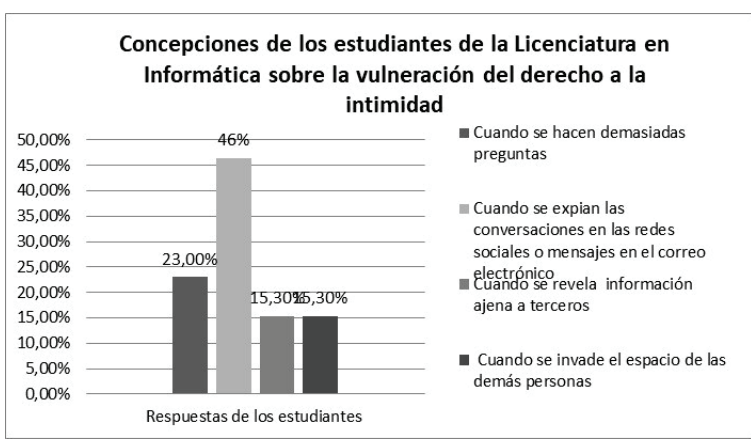

Figura 3. Concepciones de vulneración al Derecho a la Intimidad.

\section{Conclusiones}

Posterior al análisis de la información obtenida de los instrumentos y técnicas aplicadas a los estudiantes de la Licenciatura en Informática de la Universidad de Córdoba que hicieron parte de esta investigación, se pudo identificar que la concepción de derecho a la intimidad que éstos tienen, está relacionada con el concepto de intimidad en general, término que ellos conciben como aquello perteneciente a lo privado, a la sexualidad, las relaciones íntimas y a la exhibición del cuerpo, ya sea desnudo o en ropa interior; concepción que han creado a través de sus experiencias personales y su cotidianidad, que es el resultado de la realidad objetiva y lo que cada persona reconstruye por sí misma de manera subjetiva, es decir, es la construcción de la realidad que hacen un grupo de personas en un contexto histórico dado, lo que (Moscovici, 1979) define como "representaciones sociales".

Por otra parte, se puede vislumbrar que su concepción de intimidad no se acerca a lo planteado por los teóricos que han desarrollado estudios relacionados con la intimidad, puesto que los estudiantes de informática dejan por fuera muchos aspectos que hacen parte de la intimidad de las personas, ya que cuando se habla de ella según las definición dada por (ESPAÑOLA, s.f.), (Pinsón, 1997) y (Gonzalbo, 2008), incluye todo aquello que se hace fuera de la mirada de los demás y que no se comparte con todos, sino con aquellos que son muy cercanos. Entre las cosas intimas se encuentran, el estado civil, nombre, creencias religiosas, opiniones, orientación sexual, lugar de residencia, pensamientos, recuerdos, estados de ánimo y salud, entre otras que no tienen que ser del conocimiento público.

Entonces, se puede afirmar que debido a la concepción que poseen de intimidad y a que manifiestan desconocimiento de lo que es el derecho a la intimidad y de la reglamentación que protegen dicho derecho, por supuesta falta de información o por no haber tenido la necesidad de indagar sobre el mismo, ellos protegen la información que consideran "intima" en la red social Facebook, prueba de ello es que no se encontraron publicaciones en sus páginas en las cuales se pudieran observar imágenes con contenido sexual 0 fotografías en las que estos jóvenes exhibieran su cuerpo, pero sí fue posible identificar información personal considerada como intima en sus perfiles, tales como, su lugar de residencia, números de teléfono, ubicación en tiempo real, ausencias de casa, entre otros.

Asimismo cabe resaltar, que estos jóvenes al no concebir la intimidad como un derecho, desconocen los mecanismos para protestar y defender el derecho a la intimidad que protege todos estos aspectos 
considerados como parte de la vida íntima de las personas, además reconocer que los otros también gozan de este derecho y evitar publicar en sus perfiles fotografías, datos e información de terceros sin previa autorización y sin primero cuestionarse si de verdad es seguro y necesario subir toda esta información a la internet.

Es importante aclarar que todas las personas son libres de publicar la información concerniente a ellos en las redes sociales como lo menciona (Pinsón, 1997), evitándole a el individuo una proyección social y el normal establecimiento de relaciones personales. Es precisamente el derecho a la intimidad el que se deduce de la dignidad humana como se menciona en la Sentencia C-640/10 de la Corte Constitucional de Colombia y hace alusión a conceptos como dignidad, honorabilidad, autodeterminación informativa y libre desarrollo de la personalidad.

Finalmente, es importante resaltar que existe una fortaleza en los estudiantes y es la aplicación de las concepciones que poseen de intimidad y de derecho a la intimidad en la red social Facebook, es decir aplican lo que conocen y si reciben la información y capacitación correspondiente relacionada con el derecho a la intimidad, estos podrán aplicarlos en su totalidad y dar el uso adecuado a dicha red social y aplicarlo en todas las esferas de su vida.

\section{Referencias}

Arteaga Morejón, C.E y Ayala Yánez, J.G. (2015). Cultura visual y Comunicación: etnografía virtual sobre la foto de perfil en Facebook a tres estudiantes entre 13 y 15 años del Colegio Mena del Hierro en la ciudad de Quito. Trabajo de grado previo a la obtención del título de Comunicador Social. Carrera de Comunicación Social. Quito: UCE. 83 p.

Durán Segura, M. (2014). Conocimientos y comportamientos de los usuarios de la red social Facebook relacionados con la privacidad. Ámbitos. Revista Internacional de Comunicación, (26), ISSN: 1139-1979. Disponible en: https://www.redalyc.org/articulo.oa?id=16832539008

ESPAÑOLA, R. A. (s.f.). Diccionario de la lengua española. Obtenido de https://dle.rae.es/intimiad

Ferreyra, Julián Agustín y Baquero, Tomas (2014). Prácticas delderecho a la intimidad y de cuidado en Facebook: elabordaje de dicha tensión desde las conceptualizaciones deniños y adolescentes. VI Congreso Internacional de Investigación y Práctica Profesional en Psicología XXI Jornadas de Investigación Décimo Encuentro de Investigadores en Psicología del MERCOSUR. Facultad de Psicología Universidad de Buenos Aires, Buenos Aires.

Gonzalbo, F. E. (2008). El derecho a la privacidad. Cuadernos de Transparencia. México: IFAI.

Linne, Joaquín. (2016). La "multimidad": performances íntimas en Facebook de adolescentes de Buenos Aires. Estudios sociológicos, 34(100), 65-84. Recuperado en 01 de abril de 2021, de http://www.scielo.org.mx/scielo.php?script=sci_arttext \&pid=S2448-64422016000100065\&Ing=es\&tIng=es.

Moscovici, S. (1979). El psicoanálisis, su imagen y su público. Buenos Aires: Editorial Huemul.

Pisón, J. M. (1997). Vida privada e intimidad: implicaciones y perversiones. Anuario de Filosofía del Derecho. 1314. 717-738.

Rodríguez García, L., \& Magdalena Benedito, J. (2016). Perspectiva de los jóvenes sobre seguridad y privacidad en las redes sociales. Revista ICONO14 Revista Científica De Comunicación Y Tecnologías Emergentes, 14(1), 24-49. https://doi.org/10.7195/ri14.v14i1.885

Romero Bejarano, H.J. y Herrero Gutiérrez, F.J. (2015). La asunción de los peligros relacionados con la privacidad en internet y en las redes sociales por parte de los universitarios españoles. Estudio de caso aplicado a un grupo de estudiantes de la universidad de salamanca. Index.comunicaciónRevista científica en el ámbito de la Comunicación Aplicada, 5 (1), 107 121.

Salazar M. A. Z. (2014). La intimidad en los jóvenes a partir del uso de redes sociales. Folios, Revista De La Facultad De Comunicaciones, (30). Recuperado a partir de https://revistas.udea.edu.co/index.php/folios/article/vi ew/20779

Tello Díaz, L. (2013). Intimacy and «extimacy» in social networks. Ethical boundaries of facebook. [Intimidad y «extimidad» en las redes sociales. Las demarcaciones éticas de Facebook]. Comunicar, 41, 205-213. https://doi.org/10.3916/C41-2013-20

Thompson, A. G. (1992). Teachers' beliefs and conceptions: A synthesis of the research. In D. A. Grouws (Ed.), Handbook of research on mathematics teaching and learning: A project of the National Council of Teachers of Mathematics (p. 127-146). Macmillan Publishing Co, Inc. 